\title{
Membrane Topology of Subunit 8 Variant of Yeast Saccharomyces cerevisiae Mitochondrial ATPSynthase
}

\author{
IMADE ARTIKA
}

\author{
Department of Biochemistry, Faculty of Mathematics and Natural Sciences, Institut Pertanian Bogor, \\ Darmaga Campus, Bogor 16680, Indonesia \\ Phone/Fax: +62-251-8423267, E-mail:imart171@yahoo.com
}

\begin{abstract}
The yeast mitochondrial $\mathrm{F}_{1} \mathrm{~F}_{0}$-ATP synthase is a multisubunit complex that contains at least 17 different subunits. Subunit 8 of yeast mitochondrial ATP synthase is a hydrophobic protein of 48 amino acids encoded by the mitochondrial ATP 8 gene. There is no homologue of subunit 8 found in bacteria. Subunit 8 has three distinct domains; an N-terminal domain, a central hydrophobic domain and a C-terminal domain. Subunit 8 has been shown to adopt a transmembrane topology with the central hydrophobic domain spans the inner mitochondrial membrane once. In order to elucidate the need of subunit 8 to maintain transmembrane topology for its functioning, a severely functionally defective subunit 8 variant that has been introduced with double-charged residues within the central hydrophobic domain was analysed. A gene encoding this variant was expressed in a yeast strain lacking endogenous subunit 8 . The subunit 8 variant was then targeted into mitochondria. Following its assembly into mitochondrial ATP synthase complex, its membrane topology was determined. The results obtained showed that subunit 8 was obligatory to maintain a transmembrane topology for providing proper functioning. The transmembrane topology may be critical for subunit 8's proposed structural roles as part of the stator stalk of the mitochondrial ATP synthase complex.
\end{abstract}

Key words: membrane topology, ATP synthase, mitochondria, yeast

ATP synthase, also known as $\mathrm{F}_{0} \mathrm{~F}_{1}$-ATPase, is a large complex of $600 \mathrm{kDa}$ that uses the proton electrochemical gradient generated by the respiratory chain to catalyse ATP synthesis from ADP and Pi (Thomas et al. 2008). The yeast mitochondrial ATP synthase is a multisubunit complex composed of at least 17 subunits grouped into two sectors, a membrane-extrinsic sector $\left(\mathrm{F}_{1}\right)$ and a membrane-embedded sector $\left(\mathrm{F}_{0}\right)$. The two sectors are linked by protein stalks. The $F_{1}$ sector is comprised of five subunits $a_{3}, b_{3}, g_{1}, d_{1}$ and $e_{1}$, and is coupled to proton flux through the $\mathrm{F}_{0}$ sector. The $\mathrm{F}_{0}$ sector spans the membrane and is composed of subunits $b$, OSCP, d, e, f, g, h, i/j, k, which are encoded by nuclear genes, and subunits 6,8 and 9, which are encoded by mitochondrial genes. Some $\mathrm{F}_{0}$ subunits form a stator stalk anchored in the membrane that prevents futile rotation of mitochondrial ATP synthase subunits relative to the rotor during coupled ATP synthesis/hydrolysis (Stephens et al. 2003). In the inner mitochondrial membrane the ATP synthase complex can form a dimer (Fronzes et al. 2006).

The subunit 8 of yeast mitochondrial ATP synthase is a small hydrophobic polypeptide of 48 amino acids encoded by the ATP8 gene (Macreadie et al. 1983). Analysis of its primary structure has led to identification of three distinct domains; an N-terminal domain, a central hydrophobic domain (CHD) and a C-terminal domain (Devenish et al. 1992). The topology of subunit 8 , which is determined by unique introduced cysteine residues, indicates that its $\mathrm{N}$-terminus is located in the intermembrane space of mitochondria, whereas the C-terminus is located within the mitochondrial matrix (Stephens et al. 2000). Further analysis employing cysteinescanning mutagenesis showed that the first 14 and the last 13 amino acids were extrinsic to the lipid bilayer, indicating the existence of a 21 amino acid transmembrane spanning region (Stephens et al. 2003).

As a mitochondrially encoded protein, subunit 8 is transcribed and translated entirely within the organelle.
Subunit 8 is not present in prokaryotes, but is an additional subunit present in the mitochondrial ATP synthase of eukaryotes. This means that bacterial ATP synthase can naturally function without the presence of subunit 8 (Artika 2007). The immediate question therefore is to resolve the detailed structure and roles of this subunit in the enzyme complex. Several lines of evidence suggested that subunit 8 is part of the stator stalk in the yeast mitochondrial ATP synthase. Subunit 8 maintains close interactions with subunits b, d, f and 6, each of which has proposed roles as part of the stator stalk. No amino acid of subunit 8 directly participates in either ATP synthesis/hydrolysis or proton pumping, suggesting that subunit 8 is a structural component of the mitochondrial ATP synthase complex (Stephens et al. 2003).

In order to elucidate its detailed structure and function, an allotopic expression system for subunit 8 has been developed. Allotopic expression is the deliberate relocation of organellar genes to the nucleus and delivery of the gene products from the cytoplasm to the corresponding organelle. For allotopic expression of subunit 8 , a nuclear version of subunit 8 gene has been designed to be expressed in the nucleocytosolic system. To ensure that the cytoplasmically synthesized subunit 8 was imported into mitochondria, sequences encoding a mitochondrial signal peptide were fused to the N-terminus of the gene (Gearing et al. 1985). The allotopic expression system has been applied to study various aspects of subunit 8 molecular biology. This system has also been successfully used to express FLAG taggedsubunit 8 protein (Artika 2006). The allotopically expressed FLAG tagged-subunit 8 protein was imported into mitochondria and assembled into a functional ATP-synthasecomplex. The main purpose of the FLAG tag addition to subunit 8 protein was to enable detection of subunit 8 protein by means of an anti-FLAG tag monoclonal antibody. In the present study, a FLAG tagged-subunit 8 variant was 
employed to analyze the need for subunit 8 to maintain transmembrane topology for function.

The ability of subunit 8 to tolerate the presence of charged amino acids within its transmembrane domain is well documented. Given the unfavourable thermodynamics associated with the burial of unshielded charges within a lipid bilayer, it was proposed that the CHD of subunit 8 may not necessarily constitute a membrane-spanning region (Stephens 2000). On the other hand, its important roles as a part of the stator stalk may necessitate subunit 8 to maintain its transmembrane topology. In addition, subunit 8 is required for the maintenance of the interaction between $\mathrm{F}_{1}$ and $\mathrm{F}_{0}$ as well as correct $\mathrm{F}_{0}$ assembly (Roucou et al. 1999). It is therefore important to elucidate the obligatory role of subunit 8 to maintain transmembrane topology for its function. The present study was aimed to probe the membrane topology of subunit 8 variant having double-charged residues within the CHD. The hypothesis and strategy taken were as follows: upon introduction of double-charged residues within the CHD, subunit 8 variant may maintain the transmembrane topology or alternatively, it may loose its transmembrane topology and effectively become a peripheral membrane protein which is still able to assemble into a functional mitochondrial ATPsynthase-complex. In principal, it is possible to distinguish between a membrane peripheral protein and an integral protein as each should behave differently upon extraction with organic solvent or detergent.

\section{MATERIALS AND METHODS}

Materials. Saccharomyces cerevisiae strain M31 (atp8, mit ${ }^{\%}$, his6, ade 1 ) used was as previously desribed (Nagley et al. 1988). Strain FTC2 is strain M31 but expressing FLAG tagged-subunit 8 gene fused with a mitochondrial signal peptide (Roucou et al. 1999). Strain DF68 is a variant of strain M31 that expresses FLAG tagged-subunit 8 variant (L23D, L24D) fused with a mitochondrial signal peptide (Artika 2007).

Isolation of Mitochondria. Mitochondria were prepared using the glass bead method (Lang et al. 1977). The protein concentration of isolated mitochondria was determined using the Bio-Rad protein micro-assay procedure based on the method of Bradford (1976).

Extraction of Mitochondrial Protein. Mitochondrial proteins were extracted as follows. An aliquot containing 200 $\mathrm{mg}$ of mitochondria was centrifuged using Sorvall SM-24 rotor at $10000 \mathrm{rpm}$ for $10 \mathrm{~min}$ at $4{ }^{\circ} \mathrm{C}$. The pellet was then resuspended with $1 \mathrm{~mL}$ of appropriate extracting solution. To avoid protein degradation, protease inhibitors, phenylmethyl-sulphyl-fluoride (PMSF), para-aminobenzamidinic acid (PAB) and e-amino-n-caproic acid (e-ACA) were added to give final concentrations of $0.5 \mathrm{mM}, 5 \mathrm{mM}$ and $5 \mathrm{mM}$, respectively. The mixture was incubated with rotary shaking at $4{ }^{\circ} \mathrm{C}$ for $30 \mathrm{~min}$ followed by centrifugation using Sorvall RP100-AT rotor at $55000 \mathrm{rpm}$ for $30 \mathrm{~min}$ at $4{ }^{\circ} \mathrm{C}$. The supernatant was carefully separated from the pellet. The protein in the pellet (subsequently called the pellet protein) was solubilised by the addition of $20 \mathrm{~mL}$ of $5 \%$ (w/v) SDS and $20 \mathrm{~mL}$ of dissociation buffer $(62.5 \mathrm{mM}$ Tris $\mathrm{HCl}, 10 \%$ (v/ v) glycerol, $2 \%(\mathrm{w} / \mathrm{v}) \mathrm{SDS}, 0.00125(\mathrm{v} / \mathrm{v})$ bromophenol blue,
$5 \%(\mathrm{v} / \mathrm{v})$ b-mercaptoethanol). Unless otherwise stated, the extracted proteins in the supernatant were precipitated using the following procedures. First, $1 \mathrm{~mL}$ water was added and the mixture was briefly vortexed. Then 4 volumes $(8 \mathrm{~mL})$ of methanol was added. After briefly vortexing the mixture, 2 volumes $(4 \mathrm{~mL})$ of chloroform was added. Again the mixture was briefly vortexed followed by addition of 3 volumes $(6 \mathrm{~mL})$ of chloroform. The mixture was then vortexed and centrifuged using Sorvall SS-34 rotor at $9000 \mathrm{rpm}$ for $2 \mathrm{~min}$. After discharging the upper phase, protein was precipitated by addition of 3 volumes of methanol, vortexed and centrifuged at $9000 \mathrm{rpm}$ for $2 \mathrm{~min}$. After carefully removing the supernatant, the pellet was air dried. After drying, the protein (subsequently called supernatant protein) was solubilised by the addition of $20 \mathrm{~mL} \mathrm{5 \%} \mathrm{(w/v)} \mathrm{SDS} \mathrm{and} 20 \mathrm{~mL}$ dissociation buffer. Both the pellet and supernatant proteins were analysed by SDS-polyacrylamide-gel-electrophoresis.

Protein Analysis. SDS-polyacrylamide-gelelectrophoresis (SDS-PAGE) was performed as described by Laemmli (1970) using a dual adjustable slab gel unit. Following SDS-PAGE, the proteins were transferred to an Immobilon-P membrane (PVDF). The membrane was then incubated overnight at $4{ }^{\circ} \mathrm{C}$ in blotting solution containing primary antibody. After washing the non-bound primary antibody, the membrane was incubated for $1 \mathrm{~h}$ at room temperature in blotting solution containing secondary antibody (conjugated alkaline-phosphatase). Proteins were visualized using a Vistra alkaline-phosphatase-conjugate-substrate kit (Amersham Life Science, United Kingdom).

\section{RESULTS}

Detection of FLAG Tagged-Subunit 8 Protein and Extraction of Mitochondrial Protein with Various Solvents. A preliminary experiment was carried out to detect allotopically expressed FLAG tagged-subunit protein using the anti-FLAG monoclonal antibody following mitochondrial protein extraction using various organic solvents. Mitochondria were isolated from the FTC2 yeast strain grown on ethanol medium. Mitochondrial proteins were then extracted using various organic solvents (2 M urea, $4 \mathrm{M}$ urea, $8 \mathrm{M}$ urea and $100 \mathrm{mM}$ sodium carbonate $\mathrm{pH} 11.5)$. Both pellet and supernatant proteins were analysed by SDS-PAGE. After the transfer of protein to the PVDF membrane, the presence of subunit 8 was examined using anti-FLAG-tag monoclonal antibody as a probe.

Subunit 8 was clearly detected in all of the pellet fractions (Fig 1). However, no subunit 8 was detected in any of the supernatant fractions. At this stage, the data indicated that the allotopically expressed FLAG tagged-subunit 8 had been successfully assembled into a functional mitochondrial ATPsynthase-complex. Furthermore, upon extraction with various organic solvents, the FLAG tagged-subunit 8 could clearly be detected using the anti-FLAG M2 monoclonal antibody probe.

Membrane Topology of Wildtype Subunit 8. In order to analyze the membrane topology of the native subunit 8 , mitochondria were isolated from strain FTC2. Membrane proteins were then extracted from mitochondria to determine the extent to which wildtype subunit 8 could be extracted by 
organic solvents. Both the pellet and supernatant proteins were analysed by SDS-PAGE. After the transfer of proteins to the PVDF membrane, the membrane was cut into two pieces to separate the upper part, containing proteins of a molecular weight larger than $30 \mathrm{kDa}$, from the lower part, containing proteins of a molecular weight below $30 \mathrm{kDa}$. The upper part of the membrane was assayed with anti-subunit-a antibody to detect the presence of subunit-a (molecular weight of $58.5 \mathrm{kDa}$ ). The part of membrane containing proteins of molecular weight less than $14.3 \mathrm{kDa}$ was assayed with antiFLAG-tag monoclonal antibody to detect the presence of subunit 8 (molecular weight of $5.8 \mathrm{kDa}$ ). Blots were tested for subunit-a in order to determine the extent to which subunita of the membranous extrinsic $F_{1}$ sector could be recovered following extraction.

As shown in Fig 2, the a-subunit was generally extracted, but was not completely removed, from the membranes since it could clearly be detected in both the supernatant and pellet fractions. As expected, extraction using storage buffer (10 mM Tris-maleate, $600 \mathrm{mM}$ mannitol, 2 mM EGTA, $0.5 \mathrm{mM}$ phosphate, $\mathrm{pH}$ 6.8) released little a-subunit into the supernatant (lane 2, a panel), and subunit-a was still mainly present in the pellet fraction (lane 1, a panel). Under the same extraction conditions, no subunit 8 signal (lane 2, Y8 panel) was detected in the supernatant, and subunit 8 was found to be in the pellet fraction (lane 1, Y8 panel). Extraction using $4 \mathrm{M}$ urea released about $40 \%$ of a-subunit (lane 4 compared to lane 3, a panel). As expected, native subunit 8 was not extracted by $4 \mathrm{M}$ urea. Extraction using $8 \mathrm{M}$ urea gave similar results to that observed using $4 \mathrm{M}$ urea except that it was not clear why more a-subunit was released by extraction with urea $8 \mathrm{M}$ (band in lane 6 compared to band in lane 4, a panel). However, the a-subunit was still present in the pellet fraction (lane 5, a panel). Subunit 8 was not extracted by $8 \mathrm{M}$ urea. Membrane protein extraction was also carried out using $100 \mathrm{mM}$ sodium carbonate ( $\mathrm{pH} 11.5)$ as an extracting solvent. Similarly to that observed on urea extraction, the a-subunit was extracted (lane 8, a panel) but was not completely released. As expected, the wildtype subunit 8 was not extracted by sodium carbonate (lane $8, \mathrm{Y} 8$ panel) and a strong subunit 8 signal was detected in the pellet fraction (lane $7, \mathrm{Y} 8$ panel). Note that despite the amount of the starting material being the same for each extraction, a stronger subunit 8 signal was detected in the sample treated with sodium carbonate (lane 7, Y8 panel). The reason for this remains unclear. Finally, extraction using SDS-urea, released most of the a-subunit (lane 10, a panel). Surprisingly, some a-subunit was still present in the pellet fraction (lane 9, a panel). As expected, subunit 8 was extracted by SDS-urea (lane 10, Y8 panel) and no subunit 8 signal was detected in the pellet fraction (lane 9, Y8 panel).

Membrane Topology of Subunit 8 Variant. Strain DF68 was considered to be the most suitable strain to be used to investigate whether or not it is obligatory for subunit 8 to maintain a transmembrane topology for function. The subsequent membrane topology studies used the variant expressed in DF68.

Membrane proteins of mitochondria isolated from strain DF68 were extracted with various solvents using the same procedure as that used to extract mitochondrial membrane proteins of strain FTC2. Following membrane protein extraction, samples from both the pellet and the supernatant fractions were analysed by SDS-PAGE. The presence of the a-subunit and subunit 8 was assayed in both fractions. The results are shown in Fig 3.

Generally, the extraction pattern was similar to that obtained from the wildtype strain FTC2 expressing wildtype subunit 8. Extraction using dissociation buffer released a small amount of the a-subunit (lane 2, a panel), but the vast majority of this subunit was not extracted (lane 1, a panel). The subunit 8 variant was not extracted into the supernatant (lane 2, Y8 panel) and was still present in the pelleted fraction (lane 1, Y8 panel). Similar to what was observed with mitochondria from strain FTC2, extraction using $8 \mathrm{M}$ urea released about $50 \%$ of the a-subunit (lane 4 , a panel) with the remainder of the a-subunit still present in the pellet (lane 3, a panel). The subunit 8 variant was not extracted by $8 \mathrm{M}$ urea (lane 4 , Y 8 panel) and it was present in the pelleted fraction (lane 3, Y8 panel) upon extraction. Extraction using sodium carbonate released little a-subunit (lane 6, a panel) with the vast majority of this subunit remaining still in the pellet (lane 5, a panel). The subunit 8 variant was not extracted by sodium carbonate (lane 6, Y8 panel) and all of the subunit 8 variant signal was detected in the pellet (lane 5, Y8 panel). As expected, both the a-subunit and the subunit 8 variant were extracted by SDS-urea (lane 8, a and Y8 panels). While a small portion of a-subunit was still detected in the pelleted fraction (lane 7, a panel), little subunit 8 variant, if any, remained in the pellet (lane 7, Y8 panel).

\section{DISCUSSION}

Subunit 8 of the yeast mitochondrial-ATP-synthase is unique and intriguing. While the majority of the mitochondrialATP-synthase subunits are encoded by the nuclear genome, subunit 8 is encoded by a mitochondrial gene. Although it has been considered that subunit 8 plays various roles as part of the stator stalk, the bacterial enzyme naturally functions without the presence of subunit 8 . The immediate questions therefore are, why subunit 8 is present in the eukaryotic system and what is the main role of this subunit in the enzyme complex.

Considerable progress has been made toward understanding of the molecular biology of subunit 8 . However, many questions related to its function remain to be solved. The 48 amino acid protein has been found to have three distinct domains, an N-terminal domain, a C-terminal domain and a central hydrophobic domain (CHD). While the C-terminal domain has been shown to be crucial for subunit 8 assembly into the complex (Grasso et al. 1991), information concerning the role of the $\mathrm{N}$-terminal and the CHD is limited to the suggestion that the CHD spans the inner mitochondrial membrane, thus indicating that subunit 8 has a transmembrane topology. Detailed analyses of subunit 8 structure, topography and functionality, are still however lacking.

The present study attempted to elucidate the significant interaction of the CHD with the lipid bilayer of the inner mitochondrial membrane, as well as to analyze the need of subunit 8 to maintain a transmembrane topology. In principal, 


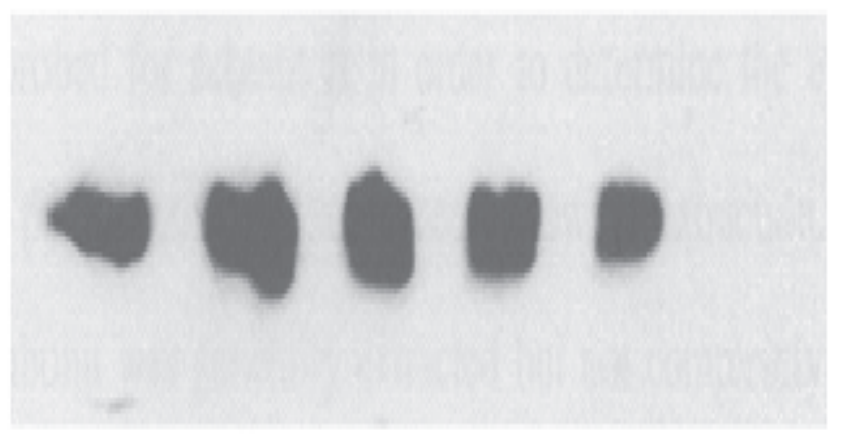

Fig 1 Detection of FLAG tagged-subunit 8 protein following extraction of mitochondrial proteins with various organic solvents and dissociation with SDS plus PVDF-membrane separation. Lane 1, pellet fraction from extraction with dissociation buffer; 2 , supernatant fraction from extraction with dissociation buffer; 3 , pellet fraction from extraction with sodium carbonate $100 \mathrm{mM}(\mathrm{pH} \mathrm{11.5);}$ 4 , supernatant fraction from extraction with sodium carbonate 100 $\mathrm{mM}(\mathrm{pH} 11.5) ; 5$, pellet fraction from extraction with $2 \mathrm{M}$ urea; 6 , supernatant fraction from extraction with $2 \mathrm{M}$ urea; 7 , pellet fraction from extraction with $4 \mathrm{M}$ urea; 8 , supernatant fraction from extraction with $4 \mathrm{M}$ urea; 9 , pellet fraction from extraction with $8 \mathrm{M}$ urea; 10 , supernatant fraction from extraction with $8 \mathrm{M}$ urea.

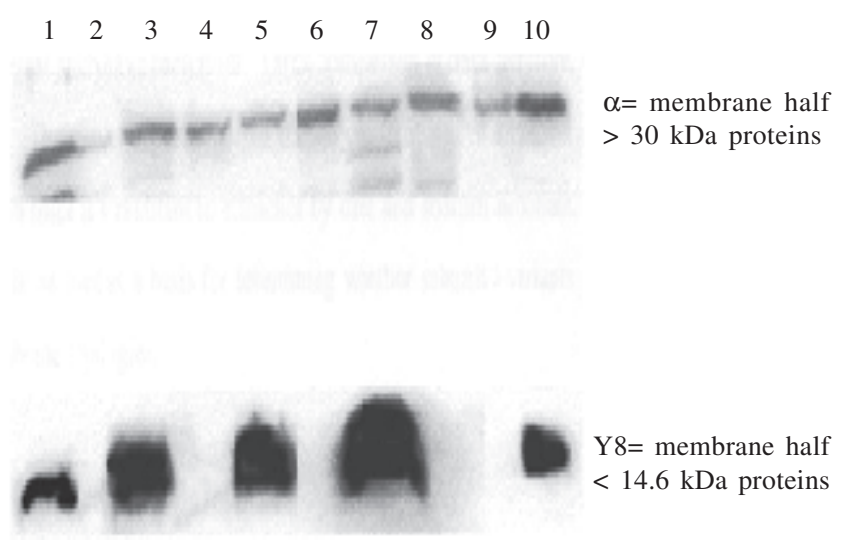

Fig 2 Extracted mitochondrial protein of wildtype strain FTC2 with various solvents and dissociated with SDS plus PVDF-membrane separation. Lane 1, pellet fraction from extraction with dissociation buffer; 2, supernatant fraction from extraction with dissociation buffer; 3 , pellet fraction from extraction with $4 \mathrm{M}$ urea; 4 , supernatant fraction from extraction with $4 \mathrm{M}$ urea; 5, pellet fraction from extraction with $8 \mathrm{M}$ urea; 6 , supernatant fraction from extraction with $8 \mathrm{M}$ urea; 7, pellet fraction from extraction with $100 \mathrm{mM}$ sodium carbonate $(\mathrm{pH} \mathrm{11.5);8}$, supernatant fraction from extraction with $100 \mathrm{mM}$ sodium carbonate ( $\mathrm{pH} \mathrm{11.5);} \mathrm{9,} \mathrm{pellet} \mathrm{fraction} \mathrm{from}$ extraction with SDS/urea; 10, supernatant fraction from extraction with SDS-urea.

the membrane spanning domain of subunit 8 can be disrupted upon the introduction of charged amino acids, because the lipid bilayer may be unable to accommodate unshielded charged residues. In the previous study (Artika 2007), six different FLAG tagged-subunit 8 variant genes, each having double-charged residues at a different position within the CHD, were allotopically expressed in a yeast strain lacking endogenous subunit 8 (strain M31). Among the functional variants, those with double-negatively-charged aspartate residues at the middle of the CHD expressed in strain DF68 was functionally the most defective. This was indicated by
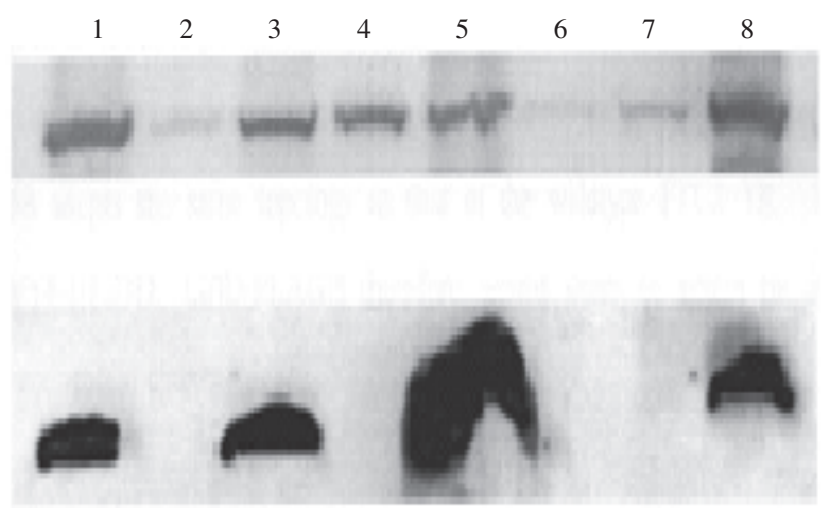

Fig 3 Extracted mitochondrial protein of strain DF68 with various solvents and dissociated with SDS plus PVDF-membrane separation. Lane 1, pellet fraction from extraction with dissociation buffer; 2, supernatant fraction from extraction with dissociation buffer; 3, pellet fraction from extraction with $8 \mathrm{M}$ urea; 4, supernatant fraction from extraction with $8 \mathrm{M}$ urea; 5, pellet fraction from extraction with $100 \mathrm{mM}$ sodium carbonate ( $\mathrm{pH} \mathrm{11.5);} \mathrm{6,} \mathrm{supernatant}$ fraction from extraction with $100 \mathrm{mM}$ sodium carbonate $(\mathrm{pH} 11.5)$; 7, pellet fraction from extraction with SDS/urea; 8, supernatant fraction from extraction with SDS-urea.

the slowest growth rate of the strain DF68 on ethanol medium. The generation time of strain DF68 is much longer $(11.1 \pm 0.5)$ compared to that of strain FTC2 $(6.9 \pm 0.4)$. For this reason, strain DF68 was employed in the present study to analyze the obligatory nature for subunit 8 of subunit 8 maintain its transmembrane topology.

Analysis of the membrane topology is based on the pattern of the protein after extraction using various solvents. As an integral protein, native subunit 8 is expected to be resistant to extraction using organic solvents. However, if the introduction of charged residues within the CHD abolishes normal transmembrane topology, it may be expected that subunit 8 would then become sensitive to organic solvent extraction. To date, no other reports on the extraction of subunit 8 by the organic solvents used in the present study exist. Direct comparison of data, therefore, can not be made. The results reported here suggest that the native subunit 8 is an integral protein since it is resistant to extraction by urea and sodium carbonate. This assumption is used as a basis for determining whether the subunit 8 variant adopts different membrane topology.

The data presented here suggest that the subunit 8 variant of strain DF68 has the same topology as the native subunit 8 protein of strain FTC2. This conclusion is based on the similarity of the protein extraction patterns of the two subunit 8 proteins. Therefore, the subunit 8 variant fully retains its integral protein character. This observation further suggests that subunit 8 must maintain its transmembrane topology, because even the most functionally defective subunit 8 variant maintains the extraction pattern of the native subunit 8 . The transmembrane topology may be critical for the proposed role of subunit 8 as part of the stator stalk of the mitochondrial ATP-synthase-complex. Both the native and variant subunit 8 proteins were resistant to extraction using organic solvents (sodium carbonate, urea), suggesting that they are integral proteins. In contrast, the peripheral $F_{1}$ a-subunit is extracted, although not completely removed, from the membrane bound-portion of the enzyme complex. As 
expected, the integral subunit 8 is only released by extraction using SDS-urea. The lipid bilayer is exquisitely sensitive to low concentration of amphipathic compounds, such as detergents that contain both polar and nonpolar groups (Manoil and Traxler 1995). In the present study, the SDSurea extraction serves as a positive control for the extraction procedures. In this case, the ability to detect subunit 8 protein from the supernatant fraction following extraction indicates that the extracted subunit 8 protein is successfully precipitated and then solubilised prior to SDS-PAGE analysis.

According to Manoil and Traxler (1995), the introduction of a single charged residue into a membrane spanning sequence is, in most cases, unlikely to change transmembrane topology. Such a substitution, however, might alter the positioning of the substituted sequence domain relative to the membrane. In DF68, two charged residues are present in the spanning sequence of subunit 8 , and the data suggest that those residues also do not change the subunit 8 transmembrane topology. Papakonstantinou et al. (1996) suggested that a differential movement of the boundaries of the putative transmembrane domain might occur following the introduction of charged residues within the CHD, such that the required hydrophobic character essential for membrane insertion, would be maintained.

Although data from the present studies have suggested that subunit 8 variant (DF68) maintains a transmembrane topology, it is necessary to confirm this finding using a different approach. One such approach is cysteine labelling. It is possible to introduce a unique cysteine residue at either $\mathrm{N}$-terminal or C-terminal end of subunit 8 . The location of the cysteine residue relative to the inner mitochondrial membrane can be examined using thiol-specific reagents as probes.

\section{ACKNOWLEDGEMENTS}

I am grateful to AusAID for sponsoring me during the course of this study. I would like to thank Rodney J. Devenish and Phillip Nagley of the Department of Biochemistry and Molecular Biology, Monash University, Victoria, Australia, for guidance and provision of facilities.

\section{REFERENCES}

Artika IM. 2006. Allotopic expression of a gene encoding FLAG tagged-subunit 8 of yeast mitochondrial ATP synthase. HAYATI J Biosci 13:36-8.

Artika IM. 2007. Structural and functional analysis of FLAG taggedsubunit 8 of yeast Saccharomyces cerevisiae mitochondrial ATP synthase. Microbiol Indones 1:33-6.
Bradford M. 1976. A rapid sensitive method for the quantities of proteins utilising the principal of protein-dye binding. Anal Biochem 72:248-54.

Devenish RJ, Papakonstantinou T, Galanis M, Law RHP, Linnane AW, Nagley P. 1992. Structure/function analysis of yeast mitochondrial ATP synthase subunit 8. Ann NY Acad Sci 671:403-14.

Fronzes R, Weimann T, Vaillier J, Velours J, Brethes D. 2006. The peripheral stalk participates in the yeast ATP synthase dimerization independently of e and g subunits. Biochemistry 45:6715-23.

Gearing DP, McMullen GL, Nagley P. 1985. Chemical synthesis of a mitochondrial gene designed for expression in the yeast nucleus. Biochem Int 10:907-15.

Grasso DG, Nero D, Law RHP, Devenish RJ, Nagley P. 1991. The Cterminal positively charged region of subunit 8 of yeast mitochondrial ATP synthase is required for efficient assembly of this subunit into the membrane $\mathrm{F}_{0}$ sector. Eur J Biochem 119:2039 .

Laemmli UK. 1970. Cleavage of structural proteins during the assembly of the head of bacteriophage T4. Nature 227:680-5.

Lang BF, Burger G, Doxiadis I, Thomas DY, Bandlow W, Kaudewitz F. 1977. A simple method for the large-scale preparation of mitochondria from microorganism. Anal Biochem 77:110-21.

Macreadie IG, Novitski CE, Maxwell RJ, John U, Ooi B, McMullen G, Lukins HB, Linnane AW, Nagley P. 1983. Biogenesis of mitochondria: the mitochondrial gene (aapl) coding for mitochondrial ATPase subunit 8 in Saccharomyces cerevisiae. Nucl Acids Res 11:4435-51.

Manoil C, Traxler B. 1995. Membrane protein assembly: genetic, evolutionary and medical perspectives. Annu Rev Genet 29:13150 .

Nagley P, Farrell LB, Gearing DP, Nero D, Meltzer S, Devenish RJ. 1988. Assembly of functional proton-translocating ATPase complex in yeast mitochondria with cytoplasmically synthesised subunit 8, a polypeptide normally encoded within the organelle. Proc Natl Acad Sci USA 85:2091-95.

Papakonstantinou T, Law RHP, Nesbitt WS, Nagley P, Devenish RJ. 1996. Molecular genetic analysis of the central hydrophobic domain of subunit 8 of yeast mitochondrial ATP synthase. Curr Genet 30:12-18.

Roucou X, Artika, IM, Devenish, RJ, Nagley P. 1999. Bioenergetic and structural consequences of allotropic expression of subunit 8 of yeast mitochondrial ATP synthase. The hydrophobic character of residues 23 and 24 is essential for maximal activity and structural stability of the enzyme complex. Eur J Biochem 261:444-51.

Stephens AN, Khan MA, Roucou X, Nagley P, Devenish RJ. 2003. The molecular neighborhood of subunit 8 of yeast mitochondrial $\mathrm{F}_{1} \mathrm{~F}_{0}$-ATP synthase probed by cysteine scanning mutagenesis and chemical modification. J Biol Chem 278:17867-75.

Stephens AN, Roucou X, Artika IM, Devenish RJ, Nagley P. 2000. Topology and proximity relationships of yeast mitochondrial ATP synthase subunit 8 determined by unique introduced cysteine residues. Eur J Biochem 267:6443-51.

Thomas D, Bron P, Weimann T, Dautant A, Giraud M, Paumard P, Salin B, Cavalier A, Velours J, Brèthes D. 2008. Supramolecular organization of the yeast $\mathrm{F}_{1} \mathrm{~F}_{0}$-ATP synthase. Biol Cell 100:591601 . 